\title{
Association of cellular and subcellular calcification with phosphorylated tau in the brains of Alzheimer's disease patients.
}

\author{
Elena Tsolaki ${ }^{1}$, Lajos Csincsik ${ }^{2}$, Jing Xue ${ }^{1}$, Imre Lengyel $^{2}$ and Sergio Bertazzo ${ }^{1 *}$
}

${ }^{1}$ Department of Medical Physics \& Biomedical Engineering, University College London, London WC1E 6BT, UK.

${ }^{2}$ Wellcome-Wolfson Institute for Experimental Medicine, School of Medicine, Dentistry and Biomedical Science, Queen's University Belfast, Belfast BT9 7BL, UK.

*To whom correspondence should be addressed: s.bertazzo@ucl.ac.uk

\begin{abstract}
Deposition of tau-tangles is an early marker for the development of Alzheimer's disease (AD). Here we show that these aggregates of phosphorylated tau protein are associated with the calcification of cell nuclei and cell projections, especially in the middle temporal, superior temporal and parahippocampal gyri. This calcification is related to the aetiology of the disease, shedding new light on the mechanisms involved in AD and potentially providing a new detection method for AD progression.
\end{abstract}


Alzheimer's disease (AD) is one of the most prevalent neurodegenerative diseases, affecting predominantly the elderly ${ }^{1}$. Hallmarks of the disease are the deposition of extracellular amyloid- $\beta(\mathrm{A} \beta)^{2,3}$ plaques and neurofibrillary tangles (NFTs) formed from hyperphosphorylated tau (pTau) $)^{3,4}$. In addition, increased mitochondrial calcium levels and the associated neuronal death ${ }^{5}$, as well as vascular intracranial calcification, have also been observed in patients with $\mathrm{AD}^{6-13}$. Here, using electron and fluorescence microscopy, we show that calcification also occurs in human brain cortical cells of AD patients at the nano and micron scale, and we hyopthesize that a potential site of calcification might be NFTs, owing to increased availability of phosphate groups and to the presence of soluble oligomerised forms of Tau protein.

We examined paraformaldehyde-fixed paraffin-embedded $4 \mu \mathrm{m}$ tissue sections from the basal ganglia at the level of the anterior commissure, temporal lobe (Brodmann areas 21 and 22) and the hippocampal region, taken from the brains of 22 AD patients, 22 age-matched elderly donors without the diagnosis of $\mathrm{AD}$, and 15 young $(<40$ years old) organ donors (Supplementary Table S1 for further information about the donors). Special attention was paid to the medial temporal lobe (middle, superior temporal and parahippocampal gyrus), a primary site of AD pathology ${ }^{14-16}$.

We detected mineralised material using backscattered electron imaging for scanning electron microscopy (SEM) (Fig. 1a, Supplementary Fig. S1) in the basal ganglia (Fig. 1aI), in the hippocampal region (Fig. 1aII) and in the temporal lobe (Fig. 1aIII), showing increased density regions in the outer layer of the caudate nucleus, the parahippocampal gyrus and the cortical area of the temporal lobe, both in AD patients and in elderly donors. Von Kossa staining (which labels the phosphates in calcium phosphates ${ }^{17}$ ) of adjacent sections (Supplementary Fig. S2), identified the same mineralised structures, suggesting the presence of calcium phosphate minerals. Interestingly, patterns of mineralisation follow the patterns of pTau immunolabelling in the cortex of AD brains ${ }^{18}$. In contrast, young donors did not present any mineralization in their brains. 

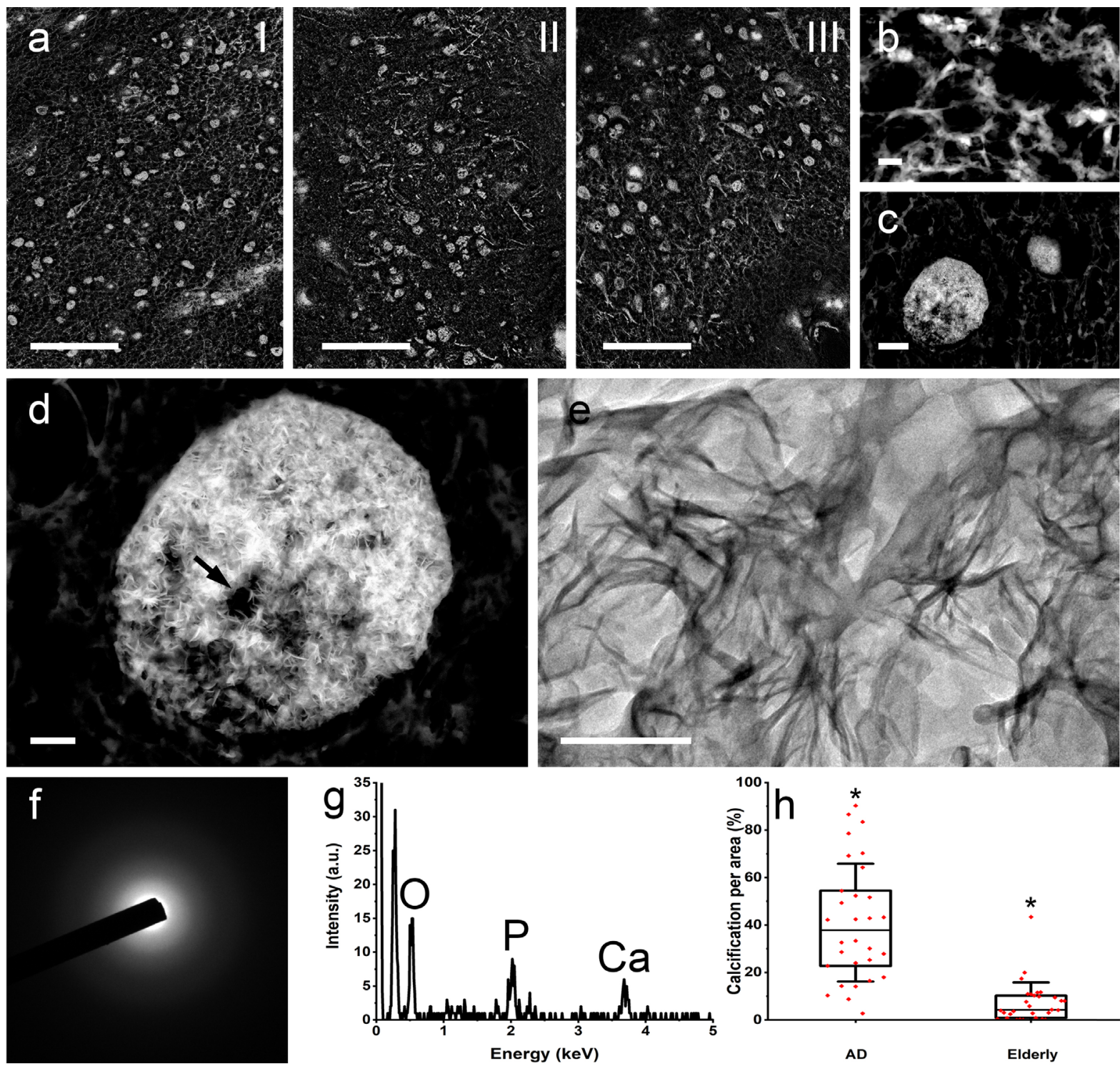

Figure 1: Calcification in histological sections of human brain. a Representative backscattered electron micrograph of basal ganglia I, hippocampal region II, and temporal lobe III. Scale bar $=100 \mu$ m. b Higher magnification image of calcified fibres. Scale bar $=2 \mu \mathrm{m}$. c Higher magnification image of nucleus-like calcification. Scale bar $=5 \mu \mathrm{m}$. d Higher magnification image of nucleus-like calcification, where the mineral is in the form of needles. Scale bar $=2 \mu \mathrm{m}$. e Transmission electron micrograph of minerals in nucleus-like calcification. Scale bar $=200 \mathrm{~nm}$. f Electron diffraction of minerals presented in e. $\mathbf{g}$ Representative EDS spectrum of all calcified structures, presenting the typical emissions for calcium and phosphorus. $\mathbf{h}$ Distribution of calcification in AD patients and in elderly organ donors. The whiskers indicate the standard deviation and the box lines the upper quartile, median, and lower quartile. $* \mathrm{p}<0.0001$.

At higher magnification, SEM micrographs show that calcification occurs in the form of fibre-like structures, resembling neuronal projections (axons and dendrites), and in the form of nucleus-like structures, resembling cell nuclei, in all brain areas (Fig. 1b, c and d). Transmission and scanning electron microscopy energy dispersive X-ray spectroscopy analysis 
and selected area electron diffraction further indicated that the mineralised structures (Fig. 1e) are formed from amorphous calcium phosphate (Fig. 1f, g). This was the case for all minerals observed, irrespective of brain region or sample group.

By quantifying the prevalence of calcified structures in the three selected brain regions, we show that $\mathrm{AD}$ patients have comparable prevalence of calcification as elderly donors (Supplementary Fig. S3a), and comparable fibre calcification, but a higher incidence of calcification in nucleus-like structures (Supplementary Fig. S3b). Importantly, calcification per tissue area is significantly higher in the brains of patients with AD $(38 \pm 24 \%)$ than in elderly donors $(4 \pm 9 \%)$ (Fig. 1h). Of note, the parahippocampal gyrus displays a high level of calcification, predominantly in the molecular, external granular and pyramidal layers of the cortex (Supplementary Fig. S4, showing fluorescently labelled samples).

We then fluorescently labelled tissue sections from a subset of $5 \mathrm{AD}$ patients and 5 elderly donors. The sections were labelled for pTau (AT8 antibody; Thermofisher MN1020) and calcification (OsteoSense 680EX; Perkin Elmer, Fig. 2 and Supplementary Fig. S5).
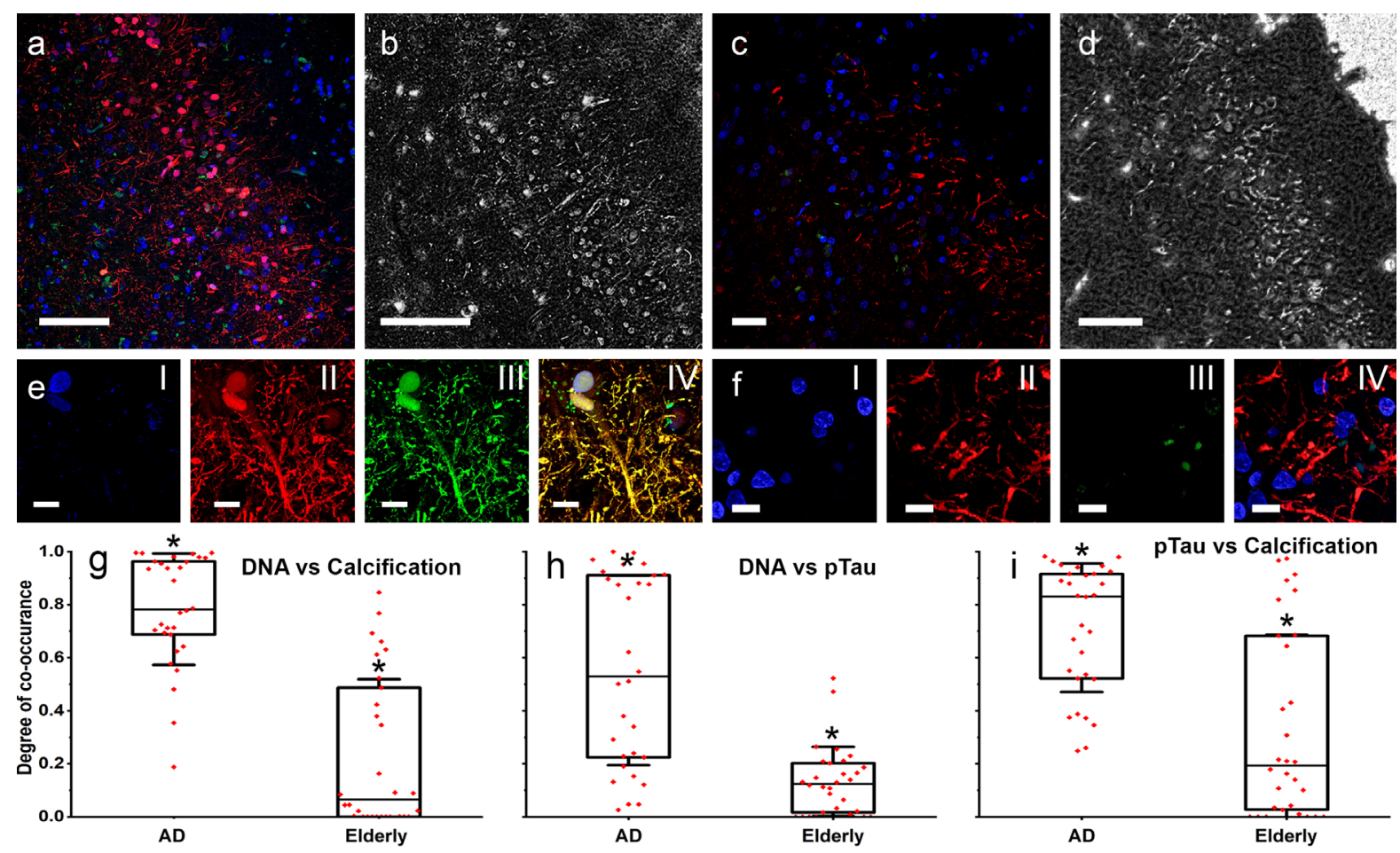

Figure 2: Fluorescence micrographs of hippocampi of AD patients and elderly donors labelled for DNA (blue), pTau (green), and calcification (red). a Fluorescence micrograph of hippocampus interface region from AD brain sample with DNA, pTau, and calcification stains. Scale bar $=77 \mu \mathrm{m}$. b Scanning electron micrograph of the same region shown in $\mathbf{a}$. Scale bar $=100 \mu \mathrm{m}$. $\mathbf{c}$ Fluorescence micrograph of hippocampus interface region from elderly organ donor brain sample with DNA, pTau and calcification stains. Scale bar $=32 \mu \mathrm{m}$. d Scanning electron micrograph of the same region shown in c. Scale bar $=50 \mu \mathrm{m}$. e High resolution fluorescence micrograph 
of DNA, pTau and calcification from AD patient brain sample. Scale bar $=14 \mu \mathrm{m}$. f High resolution fluorescence micrograph of DNA, pTau, and calcification from elderly organ donor brain sample. Scale bar $=11 \mu \mathrm{m}$. $\mathbf{g}, \mathbf{h}$ and i Statistical analysis of the co-occurrence of DAPI (DNA), OsteoSense (calcification) and pTau labelling in AD and elderly donors. The whiskers indicate the standard deviation and the box lines the upper quartile, median, and lower quartile. $* \mathrm{p}<0.0001$.

In areas with extensive calcification, the co-localisation of nuclei (labelled with DAPI, Abcam) and OsteoSense is $78 \pm 21 \%$ in $\mathrm{AD}$ patients, providing evidence that nuclear calcification is a prevalent feature in regions associated with $\mathrm{AD}$ (Fig. 2a, e, g). By contrast, co-localisation of DAPI and OsteoSense was observed in only a few of the elderly donors $(6 \pm$ 28\%) (Fig. 2c, f, g). Moreover, we found that $53 \pm 35 \%$ of pTau labelling co-localised with DAPI in AD samples (Fig. 2a, e, h), but only $12 \pm 13 \%$ in elderly donors (Fig. 2c, f, h). Finally, $83 \pm 24 \%$ of pTau labelling is co-localised with OsteoSense-labelled calcification in the AD group (Fig. 2a, e, i), but only $19 \pm 35 \%$ in samples of elderly donors (Fig. 2c, f, i).

Our results highlight that calcification of cell projections and cell nuclei are strongly associated with pTau deposition in the brains of patients with AD. Therefore, calcification may provide a new biomarker for Tau pathology in AD. Our study further suggests a potentially new mechanism in the development and/or progression of $\mathrm{AD}$, as intracellular calcification in the temporal lobe gyri would most certainly compromise cortical and hippocampal connections, leading to cognitive impairment. Apart from monitoring changes in brain, calcification could also become a pharmacological target for clinical intervention.

\section{Acknowledgements}

The study was funded through a grant (MR/N029941/1) from the National Institute for Health Research (NIHR) and the Medical Research Council (MRC) and an unrestricted grant and PhD studentship from Optos Plc.

\section{Author Contributions}

All the authors designed, conducted the experiments, interpreted the data and wrote the manuscript.

\section{References}

1 Brookmeyer, R., Johnson, E., Ziegler-Graham, K. \& Arrighi, H. M. Alzheimers Dement. 3, 186-191, (2007). 
2 Maurer, K., Riederer, P. \& Beckmann, H. Alzheimer's Disease. Epidemiology, Neuropathology, Neurochemistry, and Clinics (Springer-Verlag, 1990).

3 Lane, C. A., Hardy, J. \& Schott, J. M. Eur. J. Neurol. 25, 59-70 (2018).

4 Morris, M., Maeda, S., Vossel, K. \& Mucke, L. Neuron 70, 410-426, (2011).

5 Calvo-Rodriguez, M. et al. Nat. Comm 11, 2146, (2020).

6 Forstl, H., Burns, A., Cairns, N., Luthert, P. \& Levy, R. Behav. Neurol. 5, 53-57 (1992).

$7 \quad$ Pick, A. Am. J.Psychiatry 61, 417-436-5 (1905).

8 Förstl, H., Burns, A., Levy, R. \& Cairns, N. Br. J. Psychiatry 165, 53-59 (1994).

9 Chew, A. P. T., Gupta, G., Alatakis, S., Schneider-Kolsky, M. \& Stuckey, S. L. Radiology 265, 504-510 (2012).

10 Kockelkoren, R. et al. PLoS One 11, e0167444-e0167444 (2016).

11 Friedland, R. P., Luxenberg, J. S. \& Koss, E. A. Int. Psychogeriatr. 2, 37-43 (1990).

12 Bos, D et al. Alzheimers Dement. 8, 5(2012).

13 Song, J. Mol. Neurodegener. 14, 28 (2019).

14 Villemagne, V. L., Doré, V., Burnham, S. C., Masters, C. L. \& Rowe, C. C. Nat. Rev. Neurol. 14, 225-236 (2018).

15 van der Kant, R., Goldstein, L. S. B. \& Ossenkoppele, R. Nat. Rev. Neurosci. 21, 21 $35,(2020)$.

16 Sengoku, R. Neuropathology 40, 22-29 (2020).

17 Puchtler H. \& Meloan S.N. Histochemistry 56, 177-185 (1978).

18 Braak, H., Alafuzoff, I., Arzberger, T., Kretzschmar, H. \& Del Tredici, K. Acta Neuropathol. 112, 389-404 (2006). 


\section{Methods}

Tissue samples: For the purposes of this study brain tissue from 22 Alzheimer's disease cases, 22 elderly donors and 15 young $(<40$ years old) donors were obtained from the Queen Square Brain Bank and the Brain Tissue Bank of the University of Edinburgh. Tissue from the basal ganglia at the level of the anterior commissure, temporal lobe (Brodmann areas 21 and 22) and hippocampal region were obtained. Brain samples were formalin fixed, paraffin embedded, cut into $4 \mu \mathrm{m}$ sections and mounted onto glass histological slides.

Scanning Electron Microscopy: For scanning electron microscopy (SEM) analysis, the paraffin wax was removed using pure xylene for two 10-minute intervals. The slides were then mounted on sample holders using double sided carbon adhesive tape, were painted using silver conductive paint and coated using a 5nm layer of carbon. A Hitatchi S-3499N, a Carl Zeiss Crossbeam and a LEO 1530 were used at accelerating voltages of 5 and $10 \mathrm{kV}$ for SEM imaging; which involved imaging using secondary electron (SE) and backscattering electron (BSE) modes. Energy dispersive X-ray spectroscopy (EDS) analysis was carried out using Oxford Instruments EDX detectors, integrated into both of the microscopes.

Focused Ion Beam: A FEI Helios NanoLab 600 DualBeam Focused Ion Beam System and a Zeiss Auriga Cross Beam were used. A micro region of the sample was coated with a Platinum layer, using currents ranging between $30 \mathrm{kV}$ and $93 \mathrm{pA}$. The cut sections were imaged at $30 \mathrm{kV}$ using the BSE detector. For transmission electron microscopy (TEM) analysis a section was cut, lifted out and attached to a TEM grid.

Transmission Electron Microscopy: FIB prepared sections were transferred to a JEOL JEM 2100Plus Transmission Electron Microscope for imaging at a voltage of 200kV.

Von Kossa staining: Deparaffinisation and rehydration of the samples took place through a sequence of xylene and ethanol washes. Incubation of the samples in a 5\% silver nitrate solution (abcam ${ }^{\circledR}$ ab150687) took place for 45 minutes with exposure to 100-Watt incandescent light. The samples were then washed and incubated for 3 minutes in a sodium thiosulphate solution obtained (abcam ${ }^{\circledR}$ ab150687). The samples were rinsed with water and incubated in nuclear fast red solution (Sigma-Aldrich N8002) for 5minutes. Samples were rinsed again with water and dehydrated using absolute ethanol before washing in xylene. A coverslip was placed above 
the tissue using DPX mounting media (Sigma-Aldrich 06522) along the edges of the sample and left to dry overnight.

Immunohistochemistry: Deparaffinisation and rehydration of the samples took place through a sequence of xylene and ethanol washes. The samples were then blocked using 1:20 goat serum diluted in a tris buffered saline solution with added $0.1 \%$ triton X-100 and $0.5 \%$ bovine serum albumin (TBT) for 1 hour, followed by two TBT washes and incubation using a pTau AT8 mouse anti- human primary antibody (Thermofisher MN1020) at a concentration of 1:100. Following, the samples were washed three times with TBT for 5 minute- intervals, and incubated with the secondary antibody (Thermofisher A-21121 and abcam ${ }^{\circledR}$ ab97239) for an hour at a dilution of 1:200. The samples were then washed and incubated with DAPI stain (abcam ${ }^{\circledR}$ ab228549) at a concentration of 1:1000 (diluted in phosphate buffered saline (PBS)) for 15 minutes. The sections were also stained with OsteoSense 680EX (PerkinElmer NEV10020EX) at a concentration of 1:10 (diluted in PBS) for 20 minutes. For mounting Fluoroshield mounting medium (abcam ${ }^{\circledR}$ ab104135) was used.

Confocal microscopy: For the fluorescence imaging an Olympus FV1000, a Leica SP5 and a Zeiss LSM 980 Airyscan confocal microscope were used.

Image and statistical analysis: Image $\mathrm{J}$ was used for all image analysis done. For all analysis, 10 images per sample were used which were taken using a x63 magnifying lens. In total 10 samples were used 5 from the elderly and 5 from the AD group. For the co-occurrence analysis the Coloc 2 function of Image $\mathbf{J}$ was used. All values are reported as median +/- standard deviation. All statistical analysis was done using OriginLab 2019 and GraphPad Prism 8.3.1 software. A Mann-Witney two tailed U test was used $(\mathrm{p}<0.05)$. 